\title{
THE GLOBAL X-RAY BURST BEHAVIOUR OF KS 1731-260
}

\author{
R. Cornelisse ${ }^{1}$
}

RESUMEN

Se presenta una revisión de las propiedades de los brotes de rayos X de tipo I del sistema transitorio KS1731-260. Estas características parecen ser prototípicas para todos los brotes de rayos $\mathrm{X}$ de tipo I y podrían explicarse por la quema estable de helio que ocurre a altas tasas de acreción de masa.

\section{ABSTRACT}

An overview is given of the type I X-ray burst properties of the transient KS 1731-260. These characteristics appear to be proto-typical for all type I X-ray bursters, and stable helium burning occuring at high mass accretion rates could explain them.

\section{Key Words: ACCRETION, ACCRETION DISCS - STARS: NEUTRON — X-RAYS: BURSTS}

The Wide Field Cameras (WFC) onboard the Xray satellite BeppoSAX have monitored the Galactic Center region every spring and autumn for six years. With their $40^{\circ} \times 40^{\circ}$ field of view, $\simeq 50 \%$ of the low mass X-ray binary (LMXB) population could be observed simultaneously. About $40 \%$ of the LMXBs show type I X-ray bursts, making the WFC ideal for the study of these flashes. Type I X-ray bursts are thought to be due to unstable $\mathrm{He}$ and/or $\mathrm{H}$ burning on a neutron star surface (see e.g, Strohmayer \& Bildsten 2004 for an overview), and in total $\simeq 2000$ bursts from 54 different sources have been observed by the WFC.

One of the most frequent X-ray bursters observed by the WFC is the transient KS 1731-260, showing 342 type I X-ray bursts before it went back into quiescence in 2001. This makes it an excellent source to follow the change in X-ray burst characteristics over a large range of persistent flux levels and to test burst theory (e.g., Fujimoto et al. 1981). According to theory, at the lowest mass accretion rates $\left(\dot{M}<2 \times 10^{-10}\right.$ $\left.\Lambda_{\odot} \mathrm{yr}^{-1}\right)$ bursts are triggered by thermally unstable $\mathrm{H}$ burning, giving rise to bursts with durations $>10$ $\mathrm{s}$ and recurrence times of days to months. At intermediate accretion rates $\left(2 \times 10^{-10}<\dot{M}<10^{-9} M_{\odot}\right.$ $\left.\mathrm{yr}^{-1}\right)$, pure He burst occurs once a day with a duration $<10 \mathrm{~s}$. At the highest accretion rates $\left(\dot{M}>10^{-9}\right.$ $\left.M_{6} \mathrm{yr}^{-1}\right)$ H-rich bursts, with durations $>10 \mathrm{~s}$, occur every several hours (Fujimoto et al. 1981).

In Fig. 1 the burst-rate as a function of persistent flux is shown for KS 1731-260. At the lowest flux levels the burst-rate increases with increasing flux, but at $F_{\text {pers }} \simeq 0.12 \mathrm{WFC} \operatorname{cts~s}^{-1} \mathrm{~cm}^{-2}$ the burst-

\footnotetext{
${ }^{1}$ School of Physics and Astronomy, Southampton University, SO17 1BJ, UK (cornelis@astro.soton.ac.uk).
}

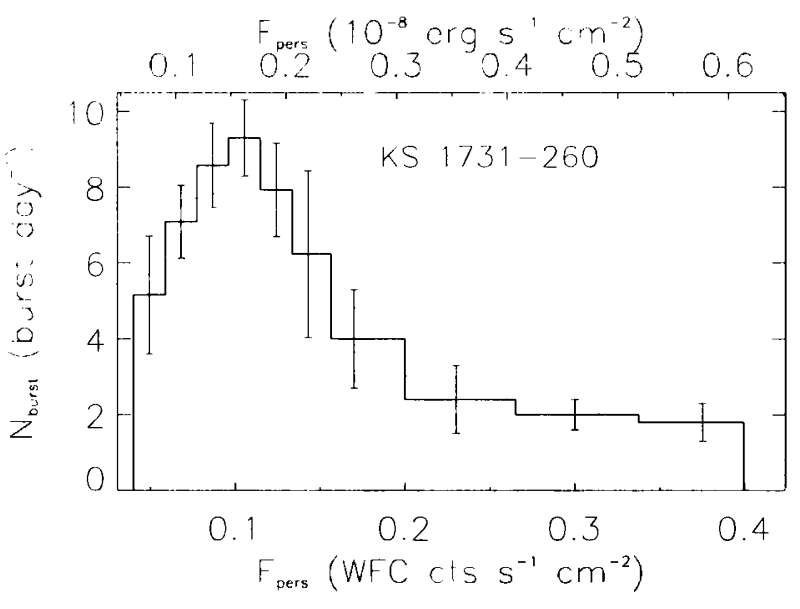

Fig. 1. Burst-rate of KS $1731-260$ as a function of observed countrate (bottom axis) and the persistent flux (top axis). From Cornelisse et al. (2003).

rate drops and levels off at the highest fluxes. Fig. 2 shows how burst durations change with persistent flux. Below $0.12 \mathrm{WFC}$ cts s $\mathrm{sm}^{-1} \mathrm{~cm}^{-2}$ bursts show a large range in duration (indicative of $\mathrm{H}$-rich bursts), while at higher fluxes the bursts appear to be $\mathrm{H}$ poor, i.e. of short duration. A similar trend is observed in Fig. 3, where at low persistent flux a linear relation is observed between the persistent flux and the time interval between X-ray bursts, while above $0.12 \mathrm{WFC}$ cts $\mathrm{s}^{-1} \mathrm{~cm}^{-2}$ this relation breaks down (note that the aliases of the linear relation in Fig. 3 are due to missed bursts during earth occulations of KS 1731-260) .

The variation of X-ray burst properties as a function of KS 1731-260 persistent flux is not unique, but appears to be universal. Cornelisse et al. (2003) showed that the nine most frequent bursters observed with the WFC have similar properties, i.e. 


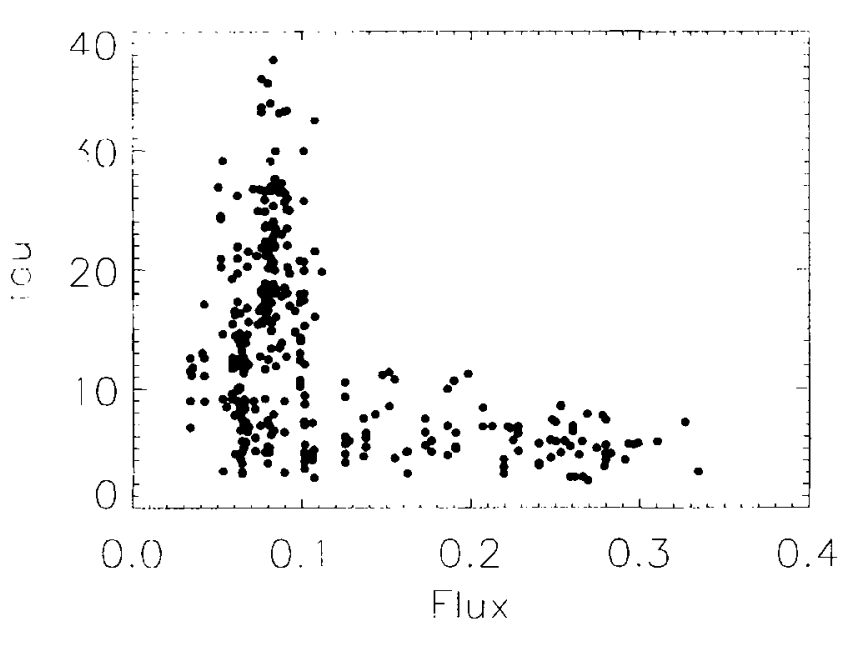

Fig. 2. Exponential decay time as a function of persistent flux for KS 1731-260 (Cornelisse et al. 2003).

there is a transition at $L_{\text {pers }} \simeq 2 \times 10^{37} \mathrm{erg} \mathrm{s}^{-1}$ from what appears to be H-rich bursts to $\mathrm{H}$-poor bursts. Curiously, 4U 1820-30, where the companion is known to be a He white dwarf, also shows a transition at this luminosity. Below $L_{\text {pers }} \simeq 2 \times 10^{37}$ erg $\mathrm{s}^{-1}$ (H-poor) X-ray bursts are observed, while above this luminosity no bursts are observed anymore.

A first interpretation could be that this is the transition from the lowest mass accretion regime to the middle regime. However, theoretically this transition is expected at luminosities 30 times lower than observed Moreover, recent simulations of the clockwork burster GS 1826-24 (the most regular burster known; Galloway et al. 2003) show that its bursts are best described if they occur in the highest mass accretion regime. These arguments indicate that at least the H-rich bursts occur in the highest mass accretion regime.

Bildsten (2000) suggested that the accreting area on the neutron star surface increases with increasing $\dot{M}$, in such a way that the accretion rate per unit area actually decreases, i.e. the burster moves from the highest accretion rate to the middle accretion rate with increasing persistent flux. However, this is very hard to reconcile with the fact that $\mathrm{H}$-rich bursts are still observed in KS 1731-260 at the lowest flux levels, and the fact that no X-ray bursts are observed in 4U $1820-30$ at high mass accretion rates.

Van Paradijs et al.(1988) already proposed that part of the fuel of X-ray bursts is burned in a stable fashion at high flux levels. This could explain the observations, since burning $\mathrm{He}$ stably produces mainly carbon, the only parameter that controls the speed at which $\mathrm{H}$ is burned (e.g., Strohmayer \& Bildsten 2004). A dramatic increase in the carbon abundance could be able to burn most $\mathrm{H}$ in the burning layer before the X-ray burst occurs, giving rise to $\mathrm{H}$-poor

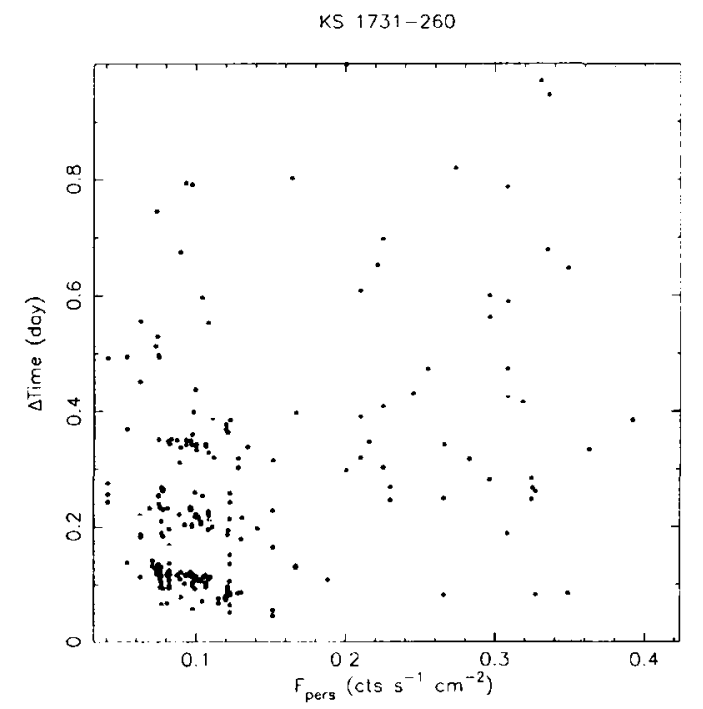

Fig. 3. Persistent $\mathrm{X}$-ray flux as a function of the time interval between bursts for KS 1731-260 (Cornelisse et al. 2003).

bursts. Furthermore, it also explains why the transition in $4 \mathrm{U} 1820-30$ is observed at a similar flux level as thise other frequent bursters. Recent theoretical simulations showed that He could be burned in a stable fashion (see e.g., Cumming 2004) at mass accretion rates below the Eddington-limit, but no good physical model exists at the moment.

In conclusion, there appears to be a universal type I X-ray burst profile that is well illustrated by the proto-typical X-ray burster KS 1731-260. A better understanding of its burst behaviour as a function of flux, will greatly help in the understanding of the physical processes that occur during type I X-ray bursts. In general the burst behaviour is well understood, but only at the highest luminosities is there a discrepancy between theory and observations. Several suggestions have been made, but a promising model is stable He burning at these luminosities.

\section{REFERENCES}

Bildsten, L. 2000, in Cosmic Explosions, eds S. Holt, \& W. Zhang, p.E65

Cornelisse, R., in 't Zand, J.J.M., Verbunt, F., et al. 2003. A\&A, 405, 1033

Cumming, A. 2004, in The Restless High Energy Universe, eds. E.P.J. van den Heuvel, J.J.M. in t Zand. \& R.A.M. Wijers, in press

Fujimoto, M. Y., Hanawa, T., \& Miyaji, S. 1981. ApJ, 247,267

Galloway, D.K., Cumming, A., Kuulkers, E.. et al. 2004. in press

van Paradijs, J., Penninx, W. \& Lewin. W.H.G. $198 \mathrm{~S}$. MNRAS, 233, 437

Strohmayer, T., \& Bildsten, L. 2004, in Compact Stellar X-ray Sources, eds. W.H.G. Le'win \& M. van der Klis. in. press 